\section{Pushing the Sanger envelope}

\section{By Matthew Mikulski, Staff Writer}

With the help of new materials, traditional Sanger sequencing could get a speed boost and still remain complementary to emerging non-Sanger techniques. In a paper published in the Proceedings of the National Academy of Sciences, researchers from Northwestern University showed that incorporating derivatives of linear polyacrylamide into a Sanger sequencing system cut DNA read times by $67 \%{ }^{1}$

Although the advance does not put the processing power of Sanger sequencing on par with newer, massively parallel sequencing technologies, the discovery would still improve the speed of a significant portion of academic research-a market that many biotech sequencing companies believe will remain Sanger-centric.

Sanger sequencing starts with multiple copies of a single strand of DNA. Complementary strands are allowed to grow on each DNA template, with special fluorescent nucleotides terminating further synthesis at various points along the templates. These single-stranded DNAs are finally run through an electrophoresis gel to determine the position of each oligonucleotide.

In the PNAS paper, the researchers built a Sanger sequencing microchip that used linear polyacrylamide (LPA) derivatives and could sequence an average of 542 nucleotide bases in 6.5 minutesalmost three times faster than a previous report of 580 bases in 18 minutes by Salas-Solano et al. using LPA. ${ }^{2}$

The single-channel sequencer described in the PNAS paper used a matrix of polydimethylacrylamide (pDMA) and a channel coating of polyhydroxyethylacrylamide (pHEA). The matrix sits in the channel and is the structure that the DNA migrates along or through when placed under an electric field.

The derivative pHEA replaced the LPA used as the electrophoresis channel coating in the Salas-Solano paper. The researchers expect that pHEA, which does not covalently bond to the channel wall, will contribute to an easier-to-use microchip-based electrophoresis system. Covalent binding of LPA can produce uneven coatings that lead to clogged channels.

Annelise Barron headed the chemical and biological engineering group at Northwestern that performed the research. She told SciBX that the next step will be to demonstrate similar performance-enhancing effects in many parallel, high-density channels. Ultimately, the group hopes to get the technology incorporated into a commercial, multichannel Sanger sequencing system.
To that end, the researchers are collaborating with Microchip Biotechnologies Inc., a company developing integrated microfluidics systems. Microchip plans to unveil its first product, the Apollo 100 integrated DNA preparation system, early this year. The system integrates all required sample preparation steps that precede an analysis using Applied Biosystems Group's capillary-based DNA sequencing machines.

Under an NIH grant, Microchip also is developing an all-in-one Sanger sequencing system in which DNA separation is carried out on a microchip. Although the technical specifications of the final product and a launch timeline have not yet been discussed, the technology behind the system was described in PNAS in May 2006. ${ }^{3}$ That paper reported a maximum sequencing read of 556 bases in 34 minutes.

If pDMA and pHEA were to be incorporated into a more commercially viable microchip design, throughput could reach up to about 8,000 bases per minute. That figure extrapolates the performance of the single channel in the PNAS paper out to 96 channels in parallel, which has previously been shown to be feasible. ${ }^{4}$

Such a tool would be a significant improvement over the current standard of capillary-based Sanger sequencing. Capillary-based systems from Applied Biosystems can achieve reads of about 550 bases in 35 minutes using the company's fastest Sanger-based technology. The company's highest-throughput product runs 96 capillaries in parallel and sequences about 1,509 bases per minute.

Barron's group has submitted a patent application covering the use of a combination of a pDMA matrix and a pHEA-adsorbed coating for microchip electrophoresis.

\section{Separation still separate}

Despite the improvements that new materials or reduced sample preparation time can bring, massively parallel sequencing solutions surpass the speed of Sanger sequencing by 100-1,000 fold. At least three companies are developing or marketing such products, including Applied Biosystems, Roche and Illumina Inc.

These next-generation products measure their performance in terms of mid to high hundreds of megabases to over one gigabase per run, with runs lasting anywhere from eight hours to eight days.

However, most companies expect that the market for Sanger sequencing will continue into the foreseeable future.

Barron noted that the amount of data generated in a single run by a massively parallel technology is not well suited for many applications of gene sequencing. For example, she said that human leukocyte antigen genotyping for organ transplants and DNA analysis in forensics are two commercial needs that demand quick reads of multiple small samples, as opposed to one huge sample processed over a day or days.

Stevan Jovanovich, president and CEO of Microchip, agreed there will always be smaller projects that don't require massively parallel sequencing. He told SciBX that almost all of the roughly $\$ 1$ billion sequencing market today will stay in the realm of Sanger applications, because researchers will continue to ask questions that can't 
be efficiently answered with massive amounts of data.

"They are checking their plasmid constructs, looking at a few hundred different genetic mutations," he noted. "Sanger is not going to go away for a while."

In addition, Barron noted that the newer massively parallel sequencing technologies have yet to become cheaper than standard capillary systems. "If you were going to sequence a viral genome of, say, 2.7 megabases, it doesn't cost less on a 454 system," she said.

Roche bought 454 Life Sciences Corp., a subsidiary of genomics company CuraGen Corp., for $\$ 140$ million in cash in March 2007. ${ }^{5}$ The business is now run out of Roche's Applied Science division.

Brendon Hill, manager of global marketing for Roche's 454 sequencing business, agreed that most of the company's next-generation systems customers will retain their Sanger instruments.

Hill thinks researchers will look more and more at larger datasets once costs go down. The inability to increase throughput and reduce costs, he said, was the reason 454 Life Sciences got out of Sanger sequencing.
“The technology has been pushed for 30 years; this paper shows a great advance, but people viewed Sanger sequencing as unable to get to the $\$ 1,000$ genome," he said.

Roche hopes to launch the successor to its Genome Sequencer FLX by year end. The new product is expected to read 1 billion bases in a day.

REFERENCES

1. Fredlake, C. et al. Proc. Nat. Acad. Sci. USA; published online Jan. 9, 2008; doi:10.1073/pnas.0705093105

Contact: Annelise E. Barron, Stanford University, Stanford, Calif. e-mail: aebarron@stanford.edu

2. Salas-Solano, O. et al. Anal. Chem. 72, 3129-3137 (2000)

3. Blazej, R. et al. Proc. Nat. Acad. Sci. USA 103, 7240-7245 (2006)

4. Paegel, B. et al. Anal. Chem. 74, 5092-5098 (2002)

5. BioCentury 15(15), B5; April 2, 2007

\section{COMPANIES AND INSTITUTIONS MENTIONED}

Applied Biosystems Group (NYSE:ABI), Foster City, Calif. CuraGen Corp. (NYSE:CRGN), Branford, Conn. Illumina Inc. (NASDAQ:ILMN), San Diego, Calif. Microchip Biotechnologies Inc., Dublin, Calif.

Northwestern University, Evanston, III.

Roche (SWX:ROG), Basel, Switzerland 